\title{
FIRST RESULTS OF A NEW ELECTROMECHANICAL CONTROLLED EXTERNAL VENTRICULAR DRAINAGE IN A PORCINE MODEL
}

\author{
Inga Margrit Elixmann ${ }^{1}$, Marian Walter ${ }^{1}$, Christine Goffin $^{1}$, Michael Czaplik ${ }^{1}$, Phil Alexander Huewelmann ${ }^{1}$, \\ Monika Kwiecien ${ }^{1}$, Martin Weinzierl ${ }^{2}$, Markus Oertel ${ }^{3}$, Wolfgang-Ingo Steudel ${ }^{4}$, Michael Kiefer ${ }^{3}$, Berno \\ Misgeld $^{1}$, Klaus Radermacher ${ }^{1}$ and Steffen Leonhardt ${ }^{1}$ \\ ${ }^{1}$ Helmholtz-Institute for Biomedical Engineering, RWTH Aachen University, Aachen, Germany \\ ${ }^{2,3,4}$ Department of Neurosurgery: ${ }^{2}$ Helios Klinikum Krefeld, Germany; ${ }^{3}$ Inselspital Bern, Switzerland; \\ ${ }^{4}$ Saarland University, Homburg, Germany \\ elixmann@hia.rwth-aachen.de
}

\begin{abstract}
Acute increase of intracranial pressure (ICP) usually has to be treated with an external ventricular drainage (EVD). Current standard mechanical EVD carry a lot of disadvantages, which hypothetically could be better managed by a newly developed electromechanical EVD. In this report our first preliminary results of such an electromechanical EVD applied in a porcine animal model are presented. The drainage was demonstrated to be both successful in monitoring and controlling elevated ICP, and able to detect slit ventricles due to overdrainage, if the indented target ICP was set too low.
\end{abstract}

Keywords: External Ventricular Drainage, Intracranial Pressure, Control, Animal Model

\section{Introduction}

An increase in intracranial volume causes raise of intracranial pressure (ICP). Such an increase can occur for example because of an imbalance of the continuous process of production and resorption of cerebrospinal fluid (CSF). Acute high ICP can be life threatening and has to be treated immediately. The standard procedure consists of insertion of a pressure sensor for ICP monitoring as well as a catheter into the ventricles for external drainage of CSF. Usually, the height of the CSF drainage reservoir determines the resulting ICP due to its hydrostatic pressure. This conventional mechanical external ventricular drainage (EVD) has potential disadvantages and possiblities of complications. If the position of the reservoir is chosen too low or the patient changes to an upright position, undesirable overdrainage may occur. The ventricles can collapse to slit ventricles and occlude the catheter perforation. Currently, there is exclusively one commercially available electromechanical EVD consisting of an ICP sensor integrated in the drainage tube followed by a peristaltic pump called Liquoguard ${ }^{\circledR}$ (Moeller Medical $\mathrm{GmbH}$ ), which is conceived of potentially minimize these problems. However, it is advisable to use this system with an additional independent ICP sensor to be able to detect slit ventricles. If slit ventricles occur, the integrated pressure sensor measures incorrect ICP values [1]. Therefore, the authors developed an innovative electromechanical "intelligent" EVD (iEVD) [2], which measures simultaneously ICP, pressure and flow in the tube and

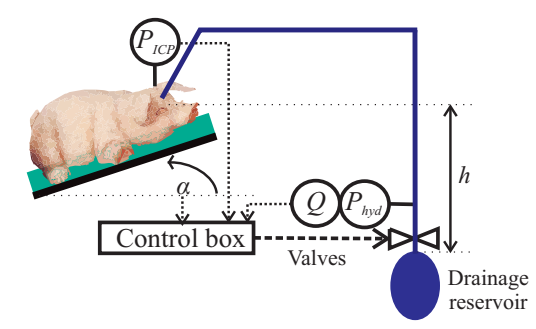

Figure 1: Experimental setup with the iEVD

patient position $\alpha$ (Figure 1). The iEVD functions by a hydrostatic pressure difference between cranium and CSF drainage reservoir and controls the ICP by positioning of a tube squeezer valve and an additional switching valve. It was already tested in an animal experiment, is able to response to changes of the drainage reservoir position or target ICP value and can indirectly detect slit ventricles.

\section{Methods}

In the present animal experiment, inducing a kaolin solution into the cisterna magna reduces the pigs's CSF resorption surface. The pig was kept under general anaesthesia. On the third day, the iEVD was implanted and tested.

The control loop with the iEVD is shown in Figure 2. The gradual tube squeezer valve sets an appropriate operating point for the system by adjusting the drainage to the target flow $Q_{\text {target }}$ and the switching valve was used in the outer control loop to keep ICP at the intended level. In recent studies, the position of the gradual tube squeezer valve was the only control variable applied. However, due to an integrative error in the position sensor of the tube squeezer valve, the nonlinear relationship of motor position to hydraulic resistance could not be compensated exactly after some time [2] and hence the switching valve was added. A filter uses a $5 \mathrm{~s}$ moving window over $100 \mathrm{~Hz}$ ICP measurements, determines the maximum (systolic) and minimum (diastolic) ICP value and calculates the average $1 \mathrm{~Hz}-\mathrm{ICP}$ value by the following equation:

$$
I C P=\frac{1}{3}\left(I C P_{\max }+2 \cdot I C P_{\min }\right)
$$

The control algorithm works with a sample frequency of $1 \mathrm{~Hz}$. At the beginning, the switching valve opens and the 
following tube squeezer valve gradually opens in minimal step size of $2.5 \mu \mathrm{m}$ until the iEVD drains a predetermined target flow $Q_{\text {target }}$. After this initialization the switching valve is controlled with a hysteresis of $0.3 \mathrm{mmHg}$. While the switching valve is open, a secondary controller increases the stored value of $Q_{\text {target }}$ by $50 \%$ if ICP is increasing more than $0.3 \mathrm{mmHg}$ within $1 \mathrm{~s}$ or ICP is not decreasing after a certain threshold time.

Whenever $Q_{E V D}<1.25 \cdot Q_{\text {target }}$, the controller increases stepwise the opening position of the tube squeezer valve until $Q_{\text {target }}$ is reached.

Slit ventricles are diagnosed when pulsation of the brain is not passed on to the water column and the difference of the maximal and minimal value within a $5 \mathrm{~s}$ window of $100 \mathrm{~Hz}$ data of the pressure sensor in the drainage tube is smaller or equal to $1 \mathrm{mmHg}$.

\section{Results}

The target ICP had to be chosen higher than $8 \mathrm{mmHg}$ otherwise the pig suffered from slit ventricles (s. Figure 3 ). With a target ICP of $8 \mathrm{mmHg}$, slit ventricles occured at approximately $t=50 \mathrm{~s}$ and at $t=56 \mathrm{~s}$ the pulsation in the water column $P_{\text {hyd }}$ got smaller than $1 \mathrm{mmHg}$ and slit ventricles were identified. The compliance of the tubing dampens the amplitude of ICP by $25 \%$.

With a higher target ICP the drainage functioned well as shown in Figure 4. At $t=110 \mathrm{~s}$ the level of the drainage reservoir was increased and the flow decreased. At $t=300 \mathrm{~s}$ the target ICP was decreased from $10.5 \mathrm{mmHg}$ to $10 \mathrm{mmHg}$ and hence $Q_{\text {target }}$ was increased by the controller to drain sufficently. The tube squeezer valve gradually opened the tube until $Q_{\text {target }}$ was reached again. After $\Delta t \approx 200 \mathrm{~s}$ the target flow was constant and only the outer control loop was active once again.

\section{Discussion}

First tests of the iEVD at a pig were successful. Potential future improvements could be an automatic increase of target pressure, if slit ventricles were detected. This preliminary iEVD can serve as a platform for more advanced control approaches: Foltz et al. [3] found out that the waveform is more reliable than mean ICP for diagnosis in humans.

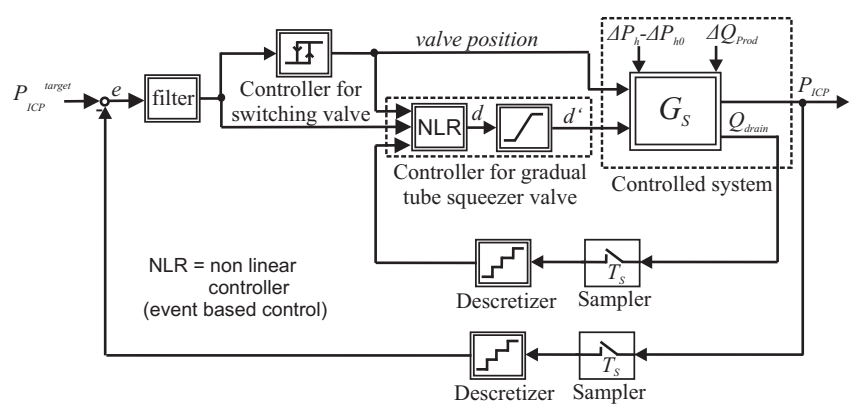

Figure 2: ICP control loop
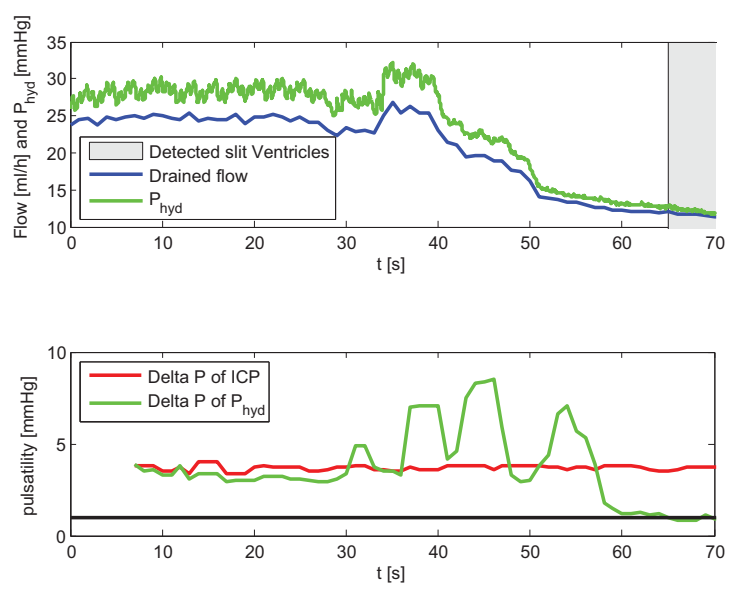

Figure 3: Slit ventricle detection
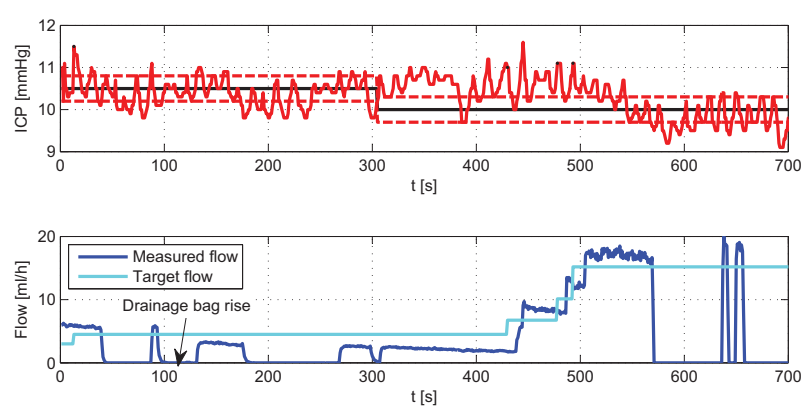

Figure 4: Measured adaption of target flow to maintain the changing target ICP at $t=300 \mathrm{~s}$

If pigs show similar single pulse waveform change following increasing ICP, it would be possible to observe how the iEVD performs in draining according to waveform.

\section{Acknowledgement}

The authors express their gratitude for the financial support of the BMBF, the support of Sensirion AG and of our project partner Reco Medizintechnik Wolfgang Rentsch e.K. and Raumedic AG.

\section{Bibliography}

[1] S. Linsler, M. Schmidtke, W. I. Steudel, M. Kiefer, and J. Oertel, "Automated intracranial pressure-controlled cerebrospinal fluid external drainage with LiquoGuard®," Acta Neurochir(Wien), Nov. 2012, Epub.

[2] I. M. Elixmann, M. Walter, C. Goffin, S. Hahne, M. Kiefer, and S. Leonhardt, "Hirndruckmodellierung und Regelung einer neuen mechatronischen externen Ventrikeldrainage," at - Automatisierungstechnik, vol. 59, pp. 613-621, Oct. 2011.

[3] E. L. Foltz and C. Aine, "Diagnosis of hydrocephalus by CSF pulse-wave analysis: a clinical study.," Surg Neurol, vol. 15, pp. 283-93, Apr. 1981. 